\title{
Moving-Horizon Estimation for Linear Dynamic Networks with Binary Encoding Schemes
}

\author{
Qinyuan Liu and Zidong Wang
}

\begin{abstract}
This paper is concerned with moving-horizon state estimation problems for a class of discrete-time linear dynamic networks. The signals are transmitted via noisy network channels and distortions can be caused by channel noises. As such, the binary encoding schemes, which take advantages of the robustness of the binary data, are exploited during the signal transmission. More specifically, under such schemes, the original signals are encoded into a bit string, transmitted via memoryless binary symmetric channels with certain crossover probabilities, and eventually restored by a decoder at the receiver. Novel centralized and decentralized moving-horizon estimators in the presence of the binary encoding schemes are constructed by solving the respective global and local least-square optimization problems. Sufficient conditions are obtained through intensive stochastic analysis to guarantee the stochastically ultimate boundedness of the estimation errors. A simulation example is presented to verify the effectiveness of the proposed moving-horizon estimators.
\end{abstract}

Index Terms-Moving-horizon estimation; Kalman filtering; remote state estimation; dynamic networks; communication constraints; binary encoding schemes.

\section{INTRODUCTION}

$\mathbf{L}$ INEAR dynamic networks are composed of a large number of nodes interconnected according to network topologies. Many complicated practical systems can be generally described by dynamic networks in terms of nodes, edges and interactions [4], [25]. Thanks to their extensive applications in diverse real-world systems (e.g. electrical power systems, manufacturing processes, compartmental systems, and biological processes), the analysis and synthesis problems of dynamic networks have become a very active research area in both industry and academia.

In recent years, considerable research efforts have been denoted to stabilization, synchronization and consensus problems of dynamic networks, see e.g. [5], [11], [23], [26]. Note

This work was supported in part by the Key Project of Science and Technology Innovation 2030 supported by the Ministry of Science and Technology of China under Grant 2018AAA0101303, the National Natural Science Foundation of China under Grants 61803282, 61873148 and 61933007, the Fundamental Research Funds for the Central Universities of China under Grant 22120180561, the "Chenguang Program" supported by Shanghai Education Development Foundation and Shanghai Municipal Education Commission of China under Grant 18CG18, and the Alexander von Humboldt Foundation of Germany. (Corresponding author: Zidong Wang)

Q. Liu is with the Department of Computer Science and Technology, Tongji University, Shanghai 201804, China; the Key Laboratory of Embedded System and Service Computing, Ministry of Education, Tongji University, Shanghai 201804, China; and the Shanghai Institute of Intelligent Science and Technology, Tongji University, Shanghai 201804, China. Email: liuqyetongji.edu. cn.

Z. Wang is with the College of Electrical Engineering and Automation, Shandong University of Science and Technology, Qingdao 266590, China, and also with the Department of Computer Science, Brunel University London, Uxbridge UB8 3PH, U.K. Email: Zidong.Wang@brunel.ac.uk. that the conventional closed-loop controller design approach usually adopts the full state-feedback techniques, thereby requiring knowledge of the full state. However, owing to the technological restrictions of the sensing devices, the full state might be unavailable in many real-world systems. A proper solution is, therefore, to make use of state estimators to extract the system state from the network measurements (usually partial observations). For this reason, the state estimation problem for dynamic networks has recently gained much research attention and a great many results have been available in the literature, see, e.g., [7], [9], [12], [27], [30], [32].

Since the pioneering work in [8], the Kalman filter has been serving as one of the most successful technologies in areas of signal processing and control engineering [14] [17], [22], [24]. A conventional Kalman filter provides the optimal estimate in the minimum mean-square error sense for linear Gauss-Markov systems. Nevertheless, unavoidable modeling errors (or parameter uncertainties) may hinder the conventional Kalman filter from being successfully applied. As such, the robust filtering problems have been investigated in the literature, see for example [33], [34], where the model perturbations are characterized by the $\tau$-divergence family and the robust Kalman filter is derived by solving a minimax problem. As the Gaussian noise assumption is necessary for a good performance of the Kalman filter, the corresponding application scope is thus limited because the noise distributions are often non-Gaussian or even unknown in many practical systems. One way to mitigate the aforementioned limitations is to utilize the so-called moving-horizon estimation strategy, whose main idea is to reconstruct the system states according to a sequence of past measurements in a moving but fixed-size window [1], [2], [6], [19], [20].

With the rapid development of communication technologies, the remote state estimation has emerged to play an important role in networked control systems [18], [21]. While the use of network equipments enjoys the benefits of flexible architecture, reduced installation and maintenance costs, some rather serious challenges do arise on the state estimation problems. For example, due to the inherent limitations of digital communication channels, the measurements transmitted from the sensor to the estimator can be affected by certain network-induced phenomena which, in turn, might result in dramatic performance degradation [29], [31]. As such, in the past decade, there has been a surge of research interest on the remote estimation problems under communication constraints with undesirable network-induced imperfection such as quantization effects [13] and multiple packet dropouts [28].

On the other hand, as pointed out in [10], the binary en- 
coding is one of the most widely employed schemes in digital communications for the reason that the binary data is both robust in transmission and simple for implementation. Under the binary encoding scheme, signals shall be first encoded into a group of binary bits and then forwarded via binary symmetric channels (BSCs). Nonetheless, the binary bits might suffer from random bit errors induced by the channel noises and, therefore, some initial efforts have been devoted to the examination on the binary encoding scheme from the perspective of information theory, see e.g. [3]. Unfortunately, when it comes to the state estimation problems, some important yet practical issues remain uninvestigated and need adequate attention. For instance, it is currently unclear 1) how to design a remote estimator that adopts encoded signals with bit errors; and 2) how to quantify the influence from the encoding scheme on the estimation performance. It is, therefore, the main objective of this paper to carry out an initial study on the moving-horizon estimation problems under binary encoding schemes.

Motivated by the above discussion, in this paper, we aim to develop moving-horizon estimation algorithms for discretetime dynamic networks under binary encoding schemes. The main contributions of this paper can be highlighted as follows. 1) The signal distortions in communication processes under the binary encoding scheme are mathematically quantified; 2) a novel framework for centralized/decentralized moving-horizon estimation is established with specific efforts to compensate the signal distortions; and 3) sufficient conditions are obtained for the stochastically ultimate boundedness of the estimation error.

Notations: The superscript ' stands for the transpose. $\operatorname{diag}\left\{A_{1}, \cdots, A_{n}\right\}$ represents a block-diagonal matrix with $A_{i}$ as the $i$ th diagonal block matrix. $\operatorname{vec}\left\{x_{1}, \cdots, x_{n}\right\}$ stacks the vectors $x_{1}, \cdots, x_{n}$ into a column vector. $\|\cdot\|$ denotes the Euclidean norm of a vector or the spectral norm of a matrix, and $\|\cdot\|_{\min }$ represents the smallest singular value of a matrix.

\section{SySTEM DESCRIPTION}

\section{A. Linear Dynamic networks}

The interconnections in dynamic networks can be described by a directed graph, denoted by $\mathcal{G} \triangleq(\mathcal{V}, \mathcal{E}, B)$, with the set of nodes $\mathcal{V}=\left\{\mathcal{S}_{1}, \mathcal{S}_{2}, \cdots, \mathcal{S}_{n}\right\}$, the set of edges $\mathcal{E}=\mathcal{V} \times \mathcal{V}$, and the weighted adjacency matrix $B=\left[b_{i j}\right]_{n \times n}$. The elements $b_{i j}$ in the weighted adjacency matrix is nonnegative and satisfy the property $b_{i j}>0 \Longleftrightarrow\left(\mathcal{S}_{i}, \mathcal{S}_{j}\right) \in \mathcal{E}$, which means the dynamics of $i$ th node can be affected by $j$ th node if and only if $b_{i j}>0$. We assume that the self-loop does not exist throughout this paper, that is, $b_{i i}=0$, for $i=1,2, \cdots, n$. The neighbors of $i$ th node is denoted by $\mathcal{N}_{i} \triangleq\left\{j \mid\left(\mathcal{S}_{i}, \mathcal{S}_{j}\right) \in \mathcal{E}\right\}$.

Consider a discrete-time linear dynamic network consisting of $n$ linearly coupled heterogeneous nodes as follows:

$$
\left\{\begin{aligned}
x_{i, k+1} & =A_{i} x_{i, k}+\sum_{j \in \mathcal{N}_{i}} b_{i j} \Gamma x_{j, k}+w_{i, k} \\
y_{i, k} & =C_{i} x_{i, k}+v_{i, k}, \text { for } i=1,2, \cdots, n
\end{aligned}\right.
$$

where $x_{i, k} \in \mathbb{R}^{n_{x}}$ and $y_{i, k} \in \mathbb{R}^{n_{y}}$ are the state vector and the measurement output of node $i . w_{i, k} \in W \subset \mathbb{R}^{n_{x}}$ and $v_{i, k} \in V \subset \mathbb{R}^{n_{y}}$ are the system noise and the measurement

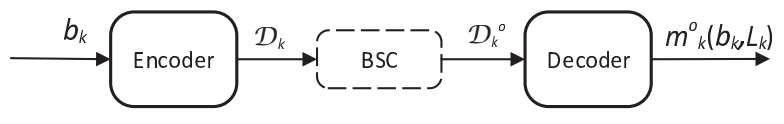

Fig. 1. The binary encoding schemes

noise of node $i$. $W$ and $V$ are bounded polyhedral sets with $0 \in W$ and $0 \in V$, respectively. The inner coupling strength $\Gamma \in \mathbb{R}^{n_{x} \times n_{x}}$ is a positive definite diagonal matrix. $A_{i}$ and $C_{i}$ are known matrices of appropriate dimensions.

\section{B. Binary encoding schemes}

As shown in Fig. 1, the binary encoding schemes are taken into consideration during signal transmission. In this case, the original signals are encoded into a finite-length binary bit string, and then transmitted to a remote estimator for further processing via a memoryless BSC.

Suppose that the range of the scalar signal $b_{k} \in \mathbb{R}$ at instant $k$ is $[-\hbar, \hbar]$, where $\hbar \in \mathbb{R}$ is an application dependent positive scalar. A binary encoder is utilized to convert the signal $b_{k}$ into a binary bit string of length $L$. Therefore, we have $2^{L}$ points denoted by $\mathcal{U} \triangleq\left\{\tau_{1}, \tau_{2}, \cdots, \tau_{2^{L}}\right\}$. These points are uniformly spaced, which divide the whole range into $2^{L}-1$ segments with a uniform interval length $\delta=\tau_{i+1}-\tau_{i}$, for $i=1,2, \cdots, 2^{L}-1$. Moreover, it can be seen that $\delta=\frac{2 \hbar}{2^{L}-1}$. Firstly, a stochastic truncation function is utilized to pre-treat the signal $b_{k}$ by $\mathcal{Q}_{k}: b_{k} \rightarrow m_{k}\left(b_{k}, L\right)$ where $m_{k}\left(b_{k}, L\right)$ is the truncated output. When $\tau_{i} \leq b_{k} \leq \tau_{i+1}$, the output $m_{k}\left(b_{k}, L\right)$ is generated according to the following probabilistic manner:

$$
\left\{\begin{array}{l}
\mathbb{P}\left\{m_{k}\left(b_{k}, L\right)=\tau_{i}\right\}=1-r_{k} \\
\mathbb{P}\left\{m_{k}\left(b_{k}, L\right)=\tau_{i+1}\right\}=r_{k}
\end{array}\right.
$$

where $r_{k} \triangleq\left(b_{k}-\tau_{i}\right) / \delta$ and $0 \leq r_{k} \leq 1$. Furthermore, the output $m_{k}\left(b_{k}, L\right)$ can be represented on a basis of binary bits: $m_{k}\left(b_{k}, L\right)=-\hbar+\sum_{i=1}^{L} h_{i, k} 2^{i-1} \delta$. Now, we have encoded $b_{k}$ into the following binary bit string:

$$
\mathcal{D}_{k} \triangleq\left\{h_{1, k}, h_{2, k}, \cdots, h_{L, k}\right\}, h_{i, k} \in\{0,1\} .
$$

The next step is to transmit the binary bit strings $\mathcal{D}_{k}$ by a memoryless BSC, where every bit might flip with a small probability (called crossover probability hereafter) because of channel noises. Accordingly, the received bit string is defined by

$$
\mathcal{D}_{k}^{o} \triangleq\left\{h_{1, k}^{o}, h_{2, k}^{o}, \cdots, h_{L, k}^{o}\right\}, h_{i, k}^{o} \in\{0,1\}
$$

where $h_{i, k}^{o}=\theta_{i, k}\left(1-h_{i, k}\right)+\left(1-\theta_{i, k}\right) h_{i, k}$ with

$$
\theta_{i, k}= \begin{cases}1, & \text { the } i \text { th bit is flipped } \\ 0, & \text { the } i \text { th bit is not flipped }\end{cases}
$$

Letting the crossover probability be $p$, we have $\mathbb{P}\left\{\theta_{i, k}=\right.$ $1\}=p$. For the convenience of analysis, we assume that $\theta_{i, k}$ are white and mutually independent in this paper. Finally, the 
received bit string $\mathcal{D}_{k}^{o}$ can be decoded to restore the original signals according to the following equation:

$$
m_{k}^{o}\left(b_{k}, L\right)=-\hbar+\sum_{i=1}^{L} h_{i, k}^{o} 2^{i-1} \delta .
$$

Owing to constrained communication bandwidth in practice, only a finite bit budget can be utilized to encode the signal over communication channels, which makes it necessary to pretreat the signals by using the truncation function. Generally speaking, under the presented binary encoding scheme, the transmission error stems mainly from two factors: the truncation errors and the flipped bit errors.

\section{Preliminary}

In this section, some preliminary knowledge of the truncation function as well as the memoryless BSC is introduced.

The output can be rewritten by

$$
m_{k}\left(b_{k}, L\right)=b_{k}+q_{k}
$$

where $q_{k} \triangleq m_{k}\left(b_{k}, L\right)-b_{k}$ represents the truncation error. According to (2), we know that $q_{k}$ is a stochastic noise obeying the Bernoulli distribution taking values at $r_{k} \delta$ or $\left(r_{k}-1\right) \delta$, i.e.,

$$
\left\{\begin{array}{l}
\mathbb{P}\left\{q_{k}=-r_{k} \delta\right\}=1-r_{k} \\
\mathbb{P}\left\{q_{k}=\left(1-r_{k}\right) \delta\right\}=r_{k}
\end{array}\right.
$$

The following lemma presents some statistical properties of the truncation error.

Lemma 1 ( [10]): The truncation error $q_{k}$ is with zero mean and bounded variance, i.e., $\mathbb{E}\left\{q_{k}\right\}=0$ and $\mathbb{E}\left\{q_{k}^{2}\right\} \leq \frac{\delta^{2}}{4}$.

An important property of BSCs is presented as follows.

Lemma 2: Assume that the signal $m_{k}\left(b_{k}, L\right)$ is transmitted via a memoryless BSC with crossover probability $p$. Then, the received signal $m_{k}^{o}\left(b_{k}, L\right)$ is with the mean and variance given by

$$
\mathbb{E}\left\{m_{k}^{o}\left(b_{k}, L\right)\right\}=(1-2 p) m_{k}\left(b_{k}, L\right)
$$

and

$$
\operatorname{Var}\left\{m_{k}^{o}\left(b_{k}, L\right)\right\}=p(1-p) \frac{4 \hbar^{2}\left(2^{2 L}-1\right)}{3\left(2^{L}-1\right)^{2}}
$$

where the expectation is taken with respect to the random variables $\theta_{i, k}$.

Proof: Taking mathematical expectation of $m_{k}^{o}\left(b_{k}, L\right)$ over the random variables $\theta_{i, k}$, we have

$$
\begin{aligned}
& \mathbb{E}\left\{m_{k}^{o}\left(b_{k}, L\right)\right\}=-\hbar+\sum_{i=1}^{L} \mathbb{E}\left\{h_{i, k}^{o}\right\} 2^{i-1} \delta \\
& =-\hbar+\sum_{i=1}^{L}\left(p\left(1-h_{i, k}\right)+(1-p) h_{i, k}\right) 2^{i-1} \delta \\
& =m_{k}\left(b_{k}, L\right)+p \sum_{i=1}^{L}\left(1-2 h_{i, k}\right) 2^{i-1} \delta
\end{aligned}
$$

where the last equality follows from $m_{k}\left(b_{k}, L\right)=-\hbar+$ $\sum_{i=1}^{L} h_{i, k} 2^{i-1} \delta$. In addition, we have

$$
\begin{aligned}
& p \sum_{i=1}^{L}\left(1-2 h_{i, k}\right) 2^{i-1} \delta=p\left(\sum_{i=1}^{L} 2^{i-1} \delta-2 \sum_{i=1}^{L} h_{i, k} 2^{i-1} \delta\right) \\
& =2 p\left(\hbar-\sum_{i=1}^{L} h_{i, k} 2^{i-1} \delta\right)=-2 p m_{k}\left(b_{k}, L\right) .
\end{aligned}
$$

Therefore, it is straightforward to verify that $\mathbb{E}\left\{m_{k}^{o}\left(b_{k}, L\right)\right\}=$ $(1-2 p) m_{k}\left(b_{k}, L\right)$. Moreover, the variance of $m_{k}^{o}\left(b_{k}, L\right)$ can be derived as follows:

$$
\begin{aligned}
& \operatorname{Var}\left\{m_{k}^{o}\left(b_{k}, L\right)\right\} \\
& =\mathbb{E}\left\{\left(-\hbar+\sum_{i=1}^{L} h_{i, k}^{o} 2^{i-1} \delta\right)^{2}\right\}-\left(\mathbb{E}\left\{m_{k}^{o}\left(b_{k}, L\right)\right\}\right)^{2} \\
& =\mathbb{E}\left\{\left(\sum_{i=1}^{L}\left(h_{i, k}^{o}-\mathbb{E}\left\{h_{i, k}^{o}\right\}\right) 2^{i-1} \delta+\mathbb{E}\left\{m_{k}^{o}\left(b_{k}, L\right)\right\}\right)^{2}\right\} \\
& -\left(\mathbb{E}\left\{m_{k}^{o}\left(b_{k}, L\right)\right\}\right)^{2} \\
& =\mathbb{E}\left\{\left(\sum_{i=1}^{L}\left(h_{i, k}^{o}-\mathbb{E}\left\{h_{i, k}^{o}\right\}\right) 2^{i-1} \delta\right)^{2}\right\} .
\end{aligned}
$$

Noting that $\theta_{i, k}$, for $i=1,2, \cdots, n$, are mutually independent, it can be seen that $h_{i, k}^{o}$ are also mutually independent which, together with the fact that $h_{i, k} \in\{0,1\}$, further indicates

$$
\begin{aligned}
& \mathbb{E}\left\{\left(\sum_{i=1}^{L}\left(h_{i, k}^{o}-\mathbb{E}\left\{h_{i, k}^{o}\right\}\right) 2^{i-1} \delta\right)^{2}\right\} \\
= & \sum_{i=1}^{L}\left(\mathbb{E}\left\{\left(h_{i, k}^{o}\right)^{2}\right\}-\left(\mathbb{E}\left\{h_{i, k}^{o}\right\}\right)^{2}\right) 2^{2 i-2} \delta^{2} \\
= & p(1-p) \frac{4 \hbar^{2}\left(2^{2 L}-1\right)}{3\left(2^{L}-1\right)^{2}} .
\end{aligned}
$$

The proof is complete now.

Up to now, we have introduced the binary encoding scheme and revealed its transmission properties for scalar signals. Furthermore, it should be emphasized that such a scheme is also applicable to the vector signals in a component-wise manner, i.e., $m_{k}\left(b_{k}, L\right)=\operatorname{vec}\left\{m_{k}\left(b_{1, k}, L\right), \cdots, m_{k}\left(b_{n, k}, L\right)\right\}$ with $b_{i, k}$ being the $i$ th entry of the vector $b_{k} \in \mathbb{R}^{n}$. For compactness, the aforementioned notations that define the scalar signals can be extended to represent the vectors. For example, when $b_{k} \in \mathbb{R}^{n}$, we have $m_{k}\left(b_{k}, L\right) \in \mathbb{R}^{n}, m_{k}^{o}\left(b_{k}, L\right) \in \mathbb{R}^{n}$ and $q_{k} \in \mathbb{R}^{n}$.

\section{Centralized Moving-Horizon Estimation}

In this section, a centralized moving-horizon estimation approach is considered. The central unit has access to the information of all the nodes. To be more specific, we assume that the local measurements $y_{i, k}$ are forwarded to the center unit using the binary encoding scheme, and then the center gives an estimate of all the plant states at each time instant.

It can be seen from Lemmas 1-2 that, compared with the original signals $y_{i, k}$, the received ones $m_{k}^{o}\left(y_{i, k}, L\right)$ are 
inevitably subject to certain degree of distortions. In order to compensate the distortions, we adopt the recovered measurements

$$
\tilde{y}_{i, k}=\gamma_{k}^{-1} m_{k}^{o}\left(y_{i, k}, L\right)
$$

where $\gamma_{k} \triangleq 1-2 p$, such that the means of the recovered signals are equal to the original ones, i.e., $\mathbb{E}\left\{\tilde{y}_{i, k}\right\}=y_{i, k}$. As a consequence, the equivalent noise stemming from the bit error in BSCs can be denoted by $\tilde{m}_{i, k} \triangleq \tilde{y}_{i, k}-m_{k}\left(y_{i, k}, L\right)$. Similar to (5), we denote the truncation error $q_{i, k} \triangleq m_{k}\left(y_{i, k}, L\right)-y_{i, k}$ and then have

$$
\tilde{y}_{i, k} \triangleq y_{i, k}+\tilde{m}_{i, k}+q_{i, k} .
$$

In what follows, we will consider the moving-horizon estimation for linear dynamic networks with binary encoding schemes. The fundamental methodology of moving-horizon estimation is to solve an optimization problem over a moving but fixed-size horizon. More specifically, at each stage $k=N, N+1, \cdots$, our purpose is to generate an estimate of all the target plants $x_{i, k-N}, \cdots, x_{i, k}(i=1,2, \cdots, n)$, denoted by $\hat{x}_{i, k-N \mid k}, \cdots, \hat{x}_{i, k \mid k}$, based on the history measurements $\tilde{y}_{i, k-N}, \cdots, \tilde{y}_{i, k}$ as well as the prediction $\bar{x}_{i, k-N}$ of the state $x_{i, k-N}(i=1,2, \cdots, n)$. A natural way of developing the prediction is to make use of the dynamical equation (1) while replacing the unknown states by the corresponding real-time estimates, i.e.,

$$
\bar{x}_{i, k-N}=A_{i} \hat{x}_{i, k-N-1 \mid k-1}+\sum_{j \in \mathcal{N}_{i}} b_{i j} \Gamma \hat{x}_{j, k-N-1 \mid k-1} .
$$

To make the formulas more compact, we introduce new notations to stack a set of vectors $v_{i, k}(i=1,2, \cdots, n)$ in the following analysis, that is, $v_{k} \triangleq \operatorname{vec}\left\{v_{1, k}, v_{2, k}, \cdots, v_{n, k}\right\}$ and $v_{k-N}^{k} \triangleq \operatorname{vec}\left\{v_{k-N}, v_{k-N+1}, \cdots, v_{k}\right\}$. Here, $v$ is generic that could be $x, y, \tilde{y}, q, \tilde{m}, v, w, \bar{x}$ or $\hat{x}$.

Now, we can state the centralized moving-horizon estimation problem as follows.

Problem 1: At each time instant $k$, for given information $\left(\bar{x}_{k-N}, \tilde{y}_{k-N}^{k}\right)$, derive the optimal state estimate $\hat{x}_{k-N \mid k}, \cdots, \hat{x}_{k \mid k}$ by minimizing a least-square cost function defined as follows:

$$
\begin{aligned}
J_{k}=\epsilon \sum_{i=1}^{n}\left\|\hat{x}_{i, k-N \mid k}-\bar{x}_{i, k-N}\right\|^{2} & \\
& +\sum_{t=k-N}^{k} \sum_{i=1}^{n}\left\|\tilde{y}_{i, t}-\hat{y}_{i, t \mid k}\right\|^{2}
\end{aligned}
$$

subject to the following constraints:

$$
\left\{\begin{aligned}
\hat{x}_{i, t+1 \mid k} & =A_{i} \hat{x}_{i, t \mid k}+\sum_{j=1}^{n} b_{i j} \Gamma \hat{x}_{j, t \mid k} \\
\hat{y}_{i, t \mid k} & =C_{i} \hat{x}_{i, t \mid k},
\end{aligned}\right.
$$

for $t=k-N, \cdots, k$, where $\epsilon$ is a positive scalar.

Remark 1: In Problem 1, the term $\left\|\hat{x}_{i, k-N \mid k}-\bar{x}_{i, k-N}\right\|^{2}$ is the initial penalty representing our beliefs in the one-step prediction, and the term $\left\|\tilde{y}_{i, t}-\hat{y}_{i, t \mid k}\right\|^{2}$ penalties the difference between the estimates and the measurements. As the one-step prediction is made on a basis of all the previous measured data, the parameter $\epsilon$ is actually utilized to weight the influence of the most recent $N$ step measured data and that of all the previous ones.

Remark 2: As stated previously, the moving-horizon estimation problems have been widely studied for linear discrete-time systems. Nevertheless, we should emphasize that the classical moving-horizon estimation strategies are no longer applicable to the underlying system in this paper since the signals suffer from signal truncation errors and stochastic bit errors induced by communication processes. These errors might dramatically degrade the estimation accuracy and, therefore, there is an urgent need to develop a novel moving-horizon estimation strategy for practical applications.

Before proceeding further, denote $\bar{A} \triangleq A+B \otimes \Gamma, A \triangleq$ $\operatorname{diag}\left\{A_{1}, \cdots, A_{n}\right\}, C \triangleq \operatorname{diag}\left\{C_{1}, \cdots, C_{n}\right\}$ and

$F_{N} \triangleq\left[\begin{array}{c}C \\ C \bar{A} \\ \vdots \\ C \overline{\bar{A}}^{N}\end{array}\right], H_{N} \triangleq\left[\begin{array}{ccccc}0 & 0 & \cdots & 0 & 0 \\ C & 0 & \cdots & 0 & 0 \\ C \bar{A} & C & \cdots & 0 & 0 \\ \vdots & \vdots & \cdots & \vdots & \vdots \\ C \bar{A}^{N-1} & C \bar{A}^{N-2} & \cdots & C \bar{A} & C\end{array}\right]$.

Utilizing these notations, we are in a position to present the following theorem.

Theorem 1: At any time $k$, for given information $\left(\bar{x}_{k-N}, \tilde{y}_{k-N}^{k}\right)$, Problem 1 has a unique optimal solution as follows

$$
\hat{x}_{k-N \mid k}=\left(\epsilon I+F_{N}^{\prime} F_{N}\right)^{-1}\left(\epsilon \bar{x}_{k-N}+F_{N}^{\prime} \tilde{y}_{k-N}^{k}\right) .
$$

Moreover, the corresponding estimation error $e_{k-N} \triangleq x_{k-N}-$ $\hat{x}_{k-N \mid k}$ is given by

$$
\begin{aligned}
e_{k-N}= & \left(\epsilon I+F_{N}^{\prime} F_{N}\right)^{-1} \\
& \times\left(\epsilon \bar{A} e_{k-N-1}+\epsilon w_{k-N-1}-F_{N}^{\prime} H_{N} w_{k-N}^{k-1}\right. \\
& \left.-F_{N}^{\prime} v_{k-N}^{k}-F_{N}^{\prime} \tilde{m}_{k-N}^{k}-F_{N}^{\prime} q_{k-N}^{k}\right) .
\end{aligned}
$$

Proof: The necessary condition on the minimum of the cost function (9) is

$$
\begin{aligned}
\nabla_{\hat{x}_{k-N \mid k}} J(k)=2 \epsilon\left(\hat{x}_{k-N \mid k}-\bar{x}_{k-N}\right) & \\
& -2 F_{N}^{\prime}\left(\tilde{y}_{k-N}^{k}-F_{N} \hat{x}_{k-N \mid k}\right)=0 .
\end{aligned}
$$

Moreover, it is easy to show that the Hessian matrix of the cost function is positive definite, and hence the cost function can be minimized by choosing the estimate as follows:

$$
\hat{x}_{k-N \mid k}=\left(\epsilon I+F_{N}^{\prime} F_{N}\right)^{-1}\left(\epsilon \bar{x}_{k-N}+F_{N}^{\prime} \tilde{y}_{k-N}^{k}\right) .
$$

Furthermore, subtracting (11) from (1) gives

$$
e_{k-N}=x_{k-N}-\left(\epsilon I+F_{N}^{\prime} F_{N}\right)^{-1}\left(\epsilon \bar{x}_{k-N}+F_{N}^{\prime} \tilde{y}_{k-N}^{k}\right) .
$$

Based on (7), the measurements can be rewritten as

$$
\tilde{y}_{k-N}^{k}=\tilde{m}_{k-N}^{k}+y_{k-N}^{k}+q_{k-N}^{k} .
$$

Substituting the one-step prediction (8) and the measurements (14) into (13) yields

$$
e_{k-N}=\left(\epsilon I+F_{N}^{\prime} F_{N}\right)^{-1}
$$




$$
\begin{aligned}
& \times\left(\epsilon \bar{A} e_{k-N-1}+\epsilon w_{k-N-1}+F_{N}^{\prime} F_{N} x_{k-N}\right. \\
& \left.-F_{N}^{\prime}\left(\tilde{m}_{k-N}^{k}+y_{k-N}^{k}+q_{k-N}^{k}\right)\right) .
\end{aligned}
$$

Noting that $y_{k-N}^{k}=F_{N} x_{k-N}+H_{N} w_{k-N}^{k-1}+v_{k-N}^{k}$, we have the dynamics of the estimation error as follows:

$$
\begin{aligned}
e_{k-N}= & \left(\epsilon I+F_{N}^{\prime} F_{N}\right)^{-1} \\
& \times\left(\epsilon \bar{A} e_{k-N-1}+\epsilon w_{k-N-1}-F_{N}^{\prime} H_{N} w_{k-N}^{k-1}\right. \\
& \left.-F_{N}^{\prime} v_{k-N}^{k}-F_{N}^{\prime} \tilde{m}_{k-N}^{k}-F_{N}^{\prime} q_{k-N}^{k}\right)
\end{aligned}
$$

which completes the proof.

The procedure of the proposed centralized moving-horizon estimation is outlined in Algorithm 1.

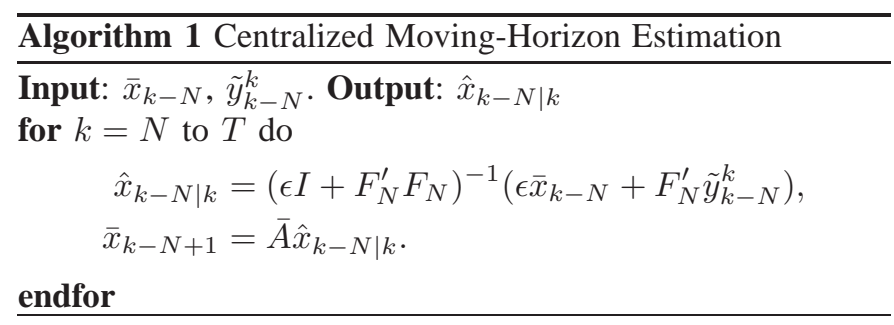

Different from the standard moving-horizon estimation proposed in [2], the stochastic behaviors of the truncation error and the flipped bit error make the estimation error (12) a stochastic sequence. As a result, we need to examine the statistical properties of the error sequence $\left\{e_{k}\right\}$. For this purpose, the following definition is introduced.

Definition 1: The error dynamics governed by (12) is said to be stochastically ultimately bounded if there exist scalars $0<\mu_{1}<1$ and $\mu_{2}>0$ such that

$$
\mathbb{E}\left\{\left\|e_{k-N}\right\|\right\} \leq \mu_{1}^{k-N} \mathbb{E}\left\{\left\|e_{0}\right\|\right\}+\mu_{2} .
$$

Next, for the sake of clarity, we denote $\bar{a} \triangleq\|\bar{A}\|, \bar{f} \triangleq$ $\left\|F_{N}\right\|, \bar{f}_{\text {min }} \triangleq\left\|F_{N}\right\|_{\min }, \bar{w} \triangleq \max _{w_{i, k} \in W}\left\|w_{i, k}\right\|, \bar{v} \triangleq$ $\max _{v_{i, k} \in V}\left\|v_{i, k}\right\|, c_{1}=\frac{\epsilon \bar{a}}{\epsilon+f_{\min }^{2}}$, and $\Delta \triangleq \frac{2 \hbar \sqrt{p(1-p)\left(2^{2 L}-1\right)}}{\sqrt{3}(1-2 p)\left(2^{L}-1\right)}$.

Now, we are ready to state the following results.

Theorem 2: The dynamics of the estimation error (12) is stochastically ultimately bounded if $0<c_{1}<1$. Moreover, the upper bound of the expectation of the estimate error can be given by

$$
\lim _{k \rightarrow \infty} \mathbb{E}\left\{\left\|e_{k}\right\|\right\} \leq \frac{c_{\infty}}{\epsilon(1-\bar{a})+\bar{f}_{\min }^{2}}
$$

where $c_{\infty}=\beta_{1}+\beta_{2} \Delta+\beta_{3} \delta, \beta_{1}=\epsilon \sqrt{n} \bar{w}+\bar{f} \bar{h} \bar{w} \sqrt{n N}+$ $\bar{f} \bar{v} \sqrt{n(N+1)}, \quad \beta_{2}=\bar{f} \sqrt{n n_{y}(N+1)}$, and $\beta_{3}=$ $0.5 \bar{f} \sqrt{n n_{y}(N+1)}$.

Proof: Taking norms to the both sides of the error dynamics (12) and exploiting triangular inequalities, we have

$$
\begin{aligned}
& \left\|e_{k-N}\right\| \leq\left\|\left(\epsilon I+F_{N}^{\prime} F_{N}\right)^{-1}\right\| \\
& \quad \times\left(\epsilon\left\|\bar{A} e_{k-N-1}\right\|+\epsilon\left\|w_{k-N-1}\right\|+\left\|F_{N}^{\prime} H_{N} w_{k-N}^{k-1}\right\|\right. \\
& \left.\quad+\left\|F_{N}^{\prime} v_{k-N}^{k}\right\|+\left\|F_{N}^{\prime} \tilde{m}_{k-N}^{k}\right\|+\left\|F_{N}^{\prime} q_{k-N}^{k}\right\|\right) .
\end{aligned}
$$

Obviously, $\left\|\left(\epsilon I+F_{N}^{\prime} F_{N}\right)^{-1}\right\|=\frac{1}{\epsilon+f_{\min }^{2}}$. Thanks to the fact that $\left\|F_{N}^{\prime} H_{N} w_{k-N}^{k-1}\right\| \leq \overline{f h} \bar{w} \sqrt{n N}^{\text {min }}$ and $\left\|F_{N}^{\prime} v_{k-N}^{k}\right\| \leq$ $\bar{f} \bar{v} \sqrt{n(N+1)}$, the following result can be derived:

$$
\begin{aligned}
\mathbb{E}\left\{\left\|e_{k-N}\right\|\right\} \leq & \frac{1}{\epsilon+\bar{f}_{\min }^{2}}\left(\epsilon \bar{a} \mathbb{E}\left\{\left\|e_{k-N-1}\right\|\right\}\right. \\
& +\epsilon \sqrt{n} \bar{w}+\bar{f} \bar{h} \bar{w} \sqrt{n N}+\bar{f} \bar{v} \sqrt{n(N+1)} \\
& \left.+\bar{f} \mathbb{E}\left\{\left\|\tilde{m}_{k-N}^{k}\right\|\right\}+\bar{f} \mathbb{E}\left\{\left\|q_{k-N}^{k}\right\|\right\}\right) .
\end{aligned}
$$

In the light of Lemmas 1-2, we can obtain that $\mathbb{E}\left\{\left\|\tilde{m}_{i, k}\right\|^{2}\right\} \leq n_{y} \Delta^{2}$ and $\mathbb{E}\left\{\left\|q_{i, k}\right\|^{2}\right\} \leq 0.25 n_{y} \delta^{2}$. Moreover, as the square root function is concave, one has from the Jensen's inequality that

$$
\mathbb{E}\left\{\left\|\tilde{m}_{k-N}^{k}\right\|\right\} \leq \sqrt{\mathbb{E}\left\{\left\|\tilde{m}_{k-N}^{k}\right\|^{2}\right\}} \leq \Delta \sqrt{n n_{y}(N+1)} .
$$

Similarly, the following inequality

$$
\mathbb{E}\left\{\left\|q_{k-N}^{k}\right\|\right\} \leq 0.5 \delta \sqrt{n n_{y}(N+1)} .
$$

holds. Substituting (17) and (18) in (16) results in $\mathbb{E}\left\{\left\|e_{k-N}\right\|\right\} \leq c_{1} \mathbb{E}\left\{\left\|e_{k-N-1}\right\|\right\}+c_{2}$, where $c_{2}=c_{\infty} /(\epsilon+$ $\left.\bar{f}_{\text {min }}^{2}\right)$. It is not difficult to prove that $\mathbb{E}\left\{\left\|e_{k-N}\right\|\right\}$ is bounded above by $\xi_{k-N}$, which is the solution to the following dynamical equation $\xi_{k-N}=c_{1} \xi_{k-N-1}+c_{2}$ with the initial condition $\xi_{0}=\mathbb{E}\left\{\left\|e_{0}\right\|\right\}$. By noting that $\xi_{k}=c_{1}^{k} \xi_{0}+\sum_{t=1}^{k} c_{1}^{t-1} c_{2}$, we can derive the following inequality

$$
\mathbb{E}\left\{\left\|e_{k-N}\right\|\right\} \leq c_{1}^{k-N} \mathbb{E}\left\{\left\|e_{0}\right\|\right\}+\frac{c_{2}}{1-c_{1}}\left(1-c_{1}^{k-N}\right)
$$

Moreover, since $0<c_{1}<1$, one has $\frac{c_{2}}{1-c_{1}}\left(1-c_{1}^{k-N}\right) \leq$ $\frac{c_{2}}{1-c_{1}}$. Therefore, it can be seen that the error dynamics (12) is stochastically uniformly bounded. Moreover, the bound (15) can be easily obtained by taking the limit of (19). The proof is complete now.

Furthermore, we have the following corollary.

Corollary 1: Suppose that the system and measurement noises are zero-mean random variables. If $0<c_{1}<1$, then the dynamics of the estimation error (12) is asymptotically stable in the mean sense, i.e.,

$$
\lim _{k \rightarrow \infty}\left\|\mathbb{E}\left\{e_{k}\right\}\right\|=0
$$

Proof: The proof follows directly from the fact that $\mathbb{E}\left\{w_{i, k}\right\}=0$ and $\mathbb{E}\left\{v_{i, k}\right\}=0$.

Remark 3: The above corollary reveals that, for the case where the system and measurement noises are zero-mean, the moving-horizon estimate will be asymptotically unbiased, thanks to the use of stochastic truncation and our dedicated efforts to compensate the signal distortions.

The proposed centralized moving-horizon estimation problem explicitly assumes that the measurements of nodes in the network should be available in the central unit for solving the optimization problem. Unfortunately, since it requires huge communication and computational capabilities, the centralized estimation is in general not practical, especially for a largescale dynamics network with lots of nodes. To handle such an issue, we will consider the decentralized moving-horizon estimation problem in the next section. 


\section{Decentralized Moving-Horizon Estimation}

In this section, we will formulate decentralized movinghorizon estimation problems. Different from the problem setup in the previous section, there does not exist a central unit in the network. The information exchange is only permitted within the neighborhood associated with the topology of the network.

The objective of decentralized moving-horizon estimation problems is to estimate the local plant states $x_{i, k-N}, \cdots, x_{i, k}$, denoted by $\hat{x}_{i, k-N \mid k}, \cdots, \hat{x}_{i, k \mid k}$, at each stage $k=N, N+$ $1, \cdots$, in a distributed manner based on the neighboring estimates $\hat{x}_{j, k-N \mid k-1}, \cdots, \hat{x}_{j, k-1 \mid k-1}\left(j \in \mathcal{N}_{i}\right)$, the local history measurements $y_{i, k-N}, \cdots, y_{i, k}$, and a prior prediction $\bar{x}_{i, k-N \mid k-1}$ of the state $x_{i, k-N}$. In this paper, the neighboring estimates are transmitted under binary encoding schemes. Consequently, the available information of $i$ th node over a finite horizon $[k-N, k]$ is given by

$$
\begin{aligned}
I_{i, k-N}^{k} \triangleq\left(y_{i, k-N}, \cdots, y_{i, k}, m_{k}^{o}\left(\hat{x}_{j, t-N \mid k-1}, L\right),\right. \\
\left.\cdots, m_{k}^{o}\left(\hat{x}_{j, k-1 \mid k-1}, L\right), j \in \mathcal{N}_{i}\right) .
\end{aligned}
$$

The local predictions $\bar{x}_{i, t \mid k-1}$ can be determined according to the dynamical equation (1) as follows:

$$
\bar{x}_{i, t \mid k-1}=A_{i} \hat{x}_{i, t-1 \mid k-1}+\sum_{j \in \mathcal{N}_{i}} b_{i j} \Gamma \tilde{x}_{j, t-1 \mid k-1}
$$

for $t=k-N, \cdots, k-1$ and $i=1,2, \cdots, n$, where $\tilde{x}_{i, t-1 \mid k-1}$ $=\gamma_{k}^{-1} m_{k}^{o}\left(\hat{x}_{i, t-1 \mid k-1}, L\right)$ is utilized to restore $\hat{x}_{j, t-1 \mid k-1}$. Accordingly, we can define the equivalent noises $\tilde{m}_{i, t-1 \mid k-1} \triangleq$ $\tilde{x}_{i, t-1 \mid k-1}-m_{k}\left(\hat{x}_{i, t-1 \mid k-1}, L\right)$. By denoting the truncation error $q_{i, t-1 \mid k-1} \triangleq-\hat{x}_{i, t-1 \mid k-1}+m_{k}\left(\hat{x}_{i, t-1 \mid k-1}, L\right)$, we have

$$
\tilde{x}_{i, t-1 \mid k-1} \triangleq \hat{x}_{i, t-1 \mid k-1}+\tilde{m}_{i, t-1 \mid k-1}+q_{i, t-1 \mid k-1} .
$$

To this end, the decentralized moving-horizon estimation problem can be presented as follows.

Problem 2: At each instant $k$, for given information $\left(\bar{x}_{i, k-N \mid k-1}, I_{i, k-N}^{k}\right)$, derive the optimal state estimate $\hat{x}_{i, k-N \mid k}, \cdots, \hat{x}_{i, k \mid k}$ by minimizing a local least-square cost function defined as follows:

$$
\begin{aligned}
J_{i}(k)=\epsilon \| \hat{x}_{i, k-N \mid k} & -\bar{x}_{i, k-N \mid k-1} \|^{2} \\
& +\sum_{t=k-N}^{k}\left\|y_{i, t}-\hat{y}_{i, t \mid k}\right\|^{2}
\end{aligned}
$$

subject to the following constraints

$$
\left\{\begin{aligned}
\hat{x}_{i, t+1 \mid k} & =A_{i} \hat{x}_{i, t \mid k}+\sum_{j \in \mathcal{N}_{i}} b_{i j} \Gamma \tilde{x}_{j, t \mid k-1} \\
\hat{y}_{i, t \mid k} & =C_{i} \hat{x}_{i, t \mid k}
\end{aligned}\right.
$$

for $t=k-N, \cdots, k$, where $\epsilon$ is a positive scalar.

Remark 4: It should be stressed that the main difference between Problems 1 and 2 lies in that the individual nodes in Problem 2 own computational capabilities, and hence are able to generate local estimates of the target plant by minimizing a local least-square cost function (23). In addition, according to the dynamical equation (1), we can see that there are complicated couplings between neighboring nodes. Consequently, to calculate $\hat{x}_{i, k-N \mid k}$, we require the exchange of the information $\hat{x}_{j, k-N \mid k-1}, \cdots, \hat{x}_{j, k-1 \mid k-1}$ within the neighborhood, which shall be transmitted through BSCs.

For a generic vector $v$, let $v_{i, k-N}^{k} \triangleq \operatorname{vec}\left\{v_{i, k-N}, \cdots\right.$, $\left.v_{i, k}\right\}$. Denote $\bar{w}_{k-N}^{k-1} \triangleq \operatorname{vec}\left\{w_{1, k-N}^{k-1}, \cdots, w_{n, k-N}^{k-1}\right\}$ and $\bar{v}_{k-N}^{k-1} \triangleq \operatorname{vec}\left\{v_{1, k-N}^{k-1}, \cdots, v_{n, k-N}^{k-1}\right\}$. Let $B_{i}$ represent the $i$ th row of the matrix $B$. The rest of the notations are defined as follows

$\bar{H}_{N} \triangleq\left[\begin{array}{cccccc}I & 0 & \cdots & 0 & 0 & 0 \\ \bar{A} & I & \cdots & 0 & 0 & 0 \\ \vdots & \vdots & \ddots & \vdots & \vdots & 0 \\ \bar{A}^{N-1} & \bar{A}^{N-2} & \cdots & \overline{\bar{A}} & I & 0\end{array}\right], \bar{F}_{N} \triangleq\left[\begin{array}{c}\bar{A} \\ \bar{A}^{2} \\ \vdots \\ \bar{A}^{N}\end{array}\right]$,

$F_{i, N} \triangleq\left[\begin{array}{c}C_{i} \\ C_{i} A_{i} \\ \vdots \\ C_{i} A_{i}^{N}\end{array}\right], H_{i, N} \triangleq\left[\begin{array}{cccc}0 & 0 & \cdots & 0 \\ C_{i} & 0 & \cdots & 0 \\ C_{i} A_{i} & C_{i} & \ddots & 0 \\ \vdots & \vdots & \ddots & \vdots \\ C_{i} A_{i}^{N-1} & C_{i} A_{i}^{N-2} & \cdots & C_{i}\end{array}\right]$,

$\widetilde{M}_{i, k-N}^{k-1} \triangleq \sum_{j \in \mathcal{N}_{i}} b_{i j} \operatorname{vec}\left\{\Gamma \tilde{x}_{j, k-N \mid k-1}, \cdots, \Gamma \tilde{x}_{j, k-1 \mid k-1}\right\}$,

$G_{i} \triangleq I_{N} \otimes\left(B_{i} \otimes \Gamma\right), \quad \mathcal{G} \triangleq \operatorname{vec}\left\{G_{1}, G_{2}, \cdots, G_{n}\right\}$,

$S_{N} \triangleq \bar{H}_{N}\left(I_{N} \otimes(B \otimes \Gamma)\right), \quad \mathcal{A}_{N} \triangleq \epsilon \bar{A}-\mathcal{F}_{N}^{\prime} \mathcal{H}_{N} \mathcal{G} \bar{F}_{N}$,

$\mathcal{H}_{N} \triangleq \operatorname{diag}\left\{H_{1, N}, H_{2, N}, \cdots, H_{n, N}\right\}, \quad \mathcal{I}_{1} \triangleq\left[\begin{array}{ll}0 & I_{N} \otimes I_{n_{x}}\end{array}\right]$, $\mathcal{F}_{N} \triangleq \operatorname{diag}\left\{F_{1, N}, F_{2, N}, \cdots, F_{n, N}\right\}, \quad \mathcal{I}_{2} \triangleq\left[\begin{array}{ll}I_{n_{x}} & 0\end{array}\right]$,

$\mathcal{W}_{N} \triangleq \mathcal{F}_{N}^{\prime} \mathcal{H}_{N} \mathcal{G}\left(S_{N}+\mathcal{I}_{1}\right)-\epsilon(B \otimes \Gamma) \mathcal{I}_{2}$.

The solution to Problem 2 is presented in the following theorem.

Theorem 3: For given information $\left(\bar{x}_{i, k-N \mid k-1}, I_{i, k-N}^{k}\right)$, Problem 2 has a unique solution given by

$$
\begin{aligned}
& \hat{x}_{i, k-N \mid k}=\left(\epsilon I+F_{i, N}^{\prime} F_{i, N}\right)^{-1} \\
& \quad \times\left(\epsilon \bar{x}_{i, k-N \mid k-1}+F_{i, N}^{\prime}\left(y_{i, k-N}^{k}-H_{i, N} \widetilde{M}_{i, k-N}^{k}\right)\right) .
\end{aligned}
$$

Moreover, denote the estimation error of $i$ th node by $e_{i, k-N} \triangleq$ $x_{i, k-N}-\hat{x}_{i, k-N \mid k}$, then the augmented error vector $e_{k-N}$ satisfies the following dynamical equation

$$
\begin{aligned}
e_{k-N}= & \left(\epsilon I+\mathcal{F}_{N}^{\prime} \mathcal{F}_{N}\right)^{-1}\left(\mathcal{A}_{N} e_{k-N-1}+\epsilon w_{k-N-1}\right. \\
& -\mathcal{F}_{N}^{\prime}\left(\mathcal{H}_{N} \bar{w}_{k-N}^{k-1}+\bar{v}_{k-N}^{k}\right)-\mathcal{F}_{N}^{\prime} \mathcal{H}_{N} \mathcal{G} \bar{H}_{N} w_{k-N-1}^{k-1} \\
& \left.+\mathcal{W}_{N}\left(\tilde{m}_{k-N-1 \mid k-1}^{k-1}+q_{k-N-1 \mid k-1}^{k-1}\right)\right) .
\end{aligned}
$$

The procedure of the proposed decentralized movinghorizon estimation is outlined in Algorithm 2.

Remark 5: Theorem 3 gives a local optimal estimate since every node has a locally known and different cost function (23). Moreover, from the results given in (25), we can see that all the exploited information, such as the local measurements $y_{i, k}$, neighboring estimates $\tilde{x}_{i, t \mid k-1}$, and the prediction $\bar{x}_{i, k-N \mid k-1}$, are available in $i$ th node, and hence the proposed distributed moving-horizon estimator is indeed applicable.

In the sequel, we will investigate the stochastically ultimate boundedness of the estimation error. First, we denote

$$
f \triangleq\left\|\mathcal{F}_{N}\right\|, f_{\min } \triangleq\left\|\mathcal{F}_{N}\right\|_{\min }, h \triangleq\left\|\mathcal{H}_{N}\right\|, \quad \bar{h} \triangleq\left\|\bar{H}_{N}\right\|
$$




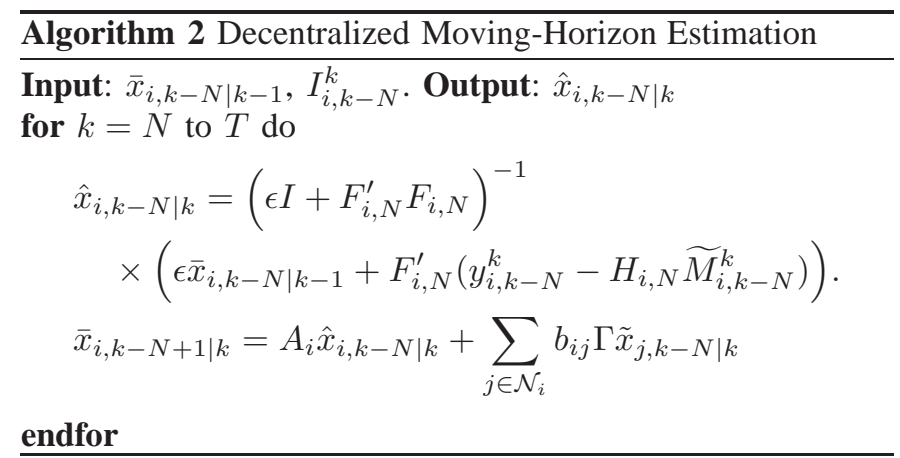

$$
g \triangleq\|\mathcal{G}\|, o \triangleq\left\|\mathcal{A}_{N}\right\|, \omega \triangleq\left\|\mathcal{W}_{N}\right\|, \quad c_{1}=\frac{o}{\epsilon+f_{\min }^{2}} .
$$

Theorem 4: The dynamics of the estimation error is stochastically ultimately bounded if $0<c_{1}<1$. Moreover, the upper bound of the expectation of the estimation error can be given by

$$
\lim _{k \rightarrow \infty} \mathbb{E}\left\{\left\|e_{k}\right\|\right\} \leq \frac{c_{\infty}}{\epsilon+f_{\min }^{2}-o}
$$

where $c_{\infty}=\beta_{1}+\beta_{2} \Delta+\beta_{3} \delta, \beta_{1}=\epsilon \bar{w} \sqrt{n}+h \bar{w} f \sqrt{n N}+$ $(\bar{v}+h g \bar{h} \bar{w}) f \sqrt{n(N+1)}, \beta_{2}=\omega \sqrt{n n_{x}(N+1)}$ and $\beta_{3}=$ $0.5 \omega \sqrt{n n_{y}(N+1)}$.

Furthermore, we have the following corollary.

Corollary 2: Suppose that the system and measurement noises are zero-mean random variables. If $0<c_{1}<1$, then the dynamics of the estimation error (26) is asymptotically stable in the mean sense, i.e., $\lim _{k \rightarrow \infty}\left\|\mathbb{E}\left\{e_{k}\right\}\right\|=0$.

Remark 6: Theorems 2-4 establish the sufficient conditions for the stochastically ultimate boundedness of the estimation error for both the centralized and the decentralized movinghorizon estimation strategies. In view of (15) and (27), we can recognize that the value of such a bound depends explicitly on the length of the bit string as well as the crossover probability.

\section{An Illustrative EXAmple}

In this section, a numerical example is presented to verify the effectiveness of the proposed centralized/decentralized moving-horizon estimation with binary encoding schemes.

The discrete-time dynamical network (1) under consideration consists of $n(n=100)$ linearly coupled nodes, which are distributed in a square region of $1 \times 1$ unit. Each node is a second-order linear system with parameters given by

$$
A_{i}=\left[\begin{array}{cc}
0.45 & 0.1 \\
0.1 & 0.3
\end{array}\right], \quad C_{i}= \begin{cases}{\left[\begin{array}{ll}
1 & 0
\end{array}\right],} & \text { for } 1 \leq i \leq 10 \\
{\left[\begin{array}{ll}
0 & 0
\end{array}\right],} & \text { for } 11 \leq i \leq n\end{cases}
$$

The topology that describes the interconnections of the coupled nodes is shown in Fig. 2. The elements of weighted adjacency matrix $B$ is set as

$$
b_{i j}=\left\{\begin{array}{lr}
0.51 \exp (-\alpha(i, j)) & \text { if } \alpha(i, j)<1 / 6 \\
0 & \text { otherwise }
\end{array}\right.
$$

where $\alpha(i, j)=\sqrt{\left(x_{i, 0}-x_{j, 0}\right)^{\prime}\left(x_{i, 0}-x_{j, 0}\right)}$. Each component of noises $w_{k}$ and $v_{i, k}$ is randomly and uniformly distributed in the respective regions $[-0.04,0.04]$ and $[-0.1,0.1]$, and

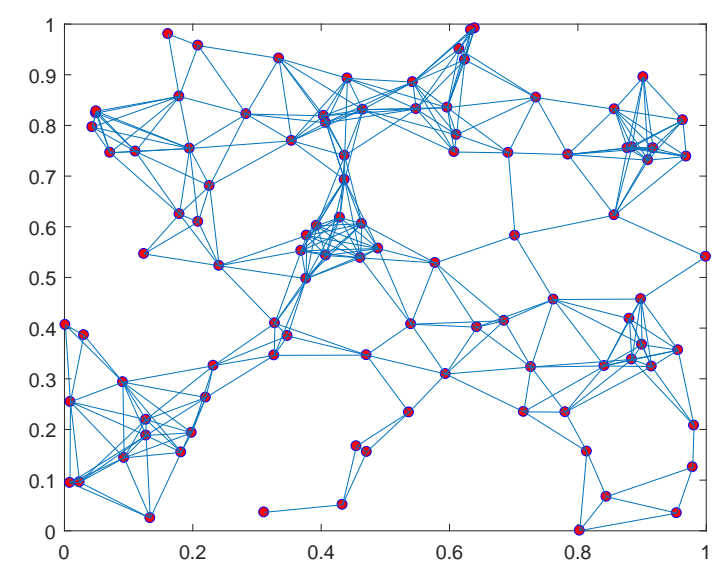

Fig. 2. The topology of the coupled network.

therefore their Euclidean norms are $\bar{w}=0.04$ and $\bar{v}=0.1$. The coupling strength is chosen to be $\Gamma=0.1 I_{2}$. Choose the size of the window $N=5$, the range $W=1.27$, the length $L=8$, the scaling weight $\epsilon=0.4$ and the interval $\delta=0.01$. The flipping probability $p$ can be identified by statistical experiments in practical applications, which is assumed to be 0.01 here. The initial estimates of the plants are given by $\hat{x}_{i, 0}=0$, for $i=1, \cdots, n$. Our objective is to derive the optimal estimate for centralized and decentralized moving-horizon estimation, respectively.

The hardware test-bed consists of a $2.10 \mathrm{GHz}$ Intel Core i7 processor with 8GB RAM running MATLAB ${ }^{\circledR} 2016 \mathrm{~B}$. We carry out $N_{M C}=100$ repeated simulations of the optimal centralized moving-horizon estimation (CMHE) based on Algorithm 1. An average estimation error is adopted to evaluated the performance of the CMHE as

$$
\mathbf{E}_{C H M E}(k)=\frac{1}{N_{M C}} \sum_{r=1}^{N_{M C}}\left(\frac{1}{n} \sum_{i=1}^{n}\left\|x_{i, k-N}^{(r)}-\hat{x}_{i, k-N \mid k}^{(r)}\right\|\right) .
$$

The superscript " $(r)$ " implies that the value is obtained in the $r$ th run. The simulation results are depicted in Fig. 3. Moreover, we compared the proposed algorithm with the centralized Kalman filter (CKF) [8], whose initial error covariance is set to be $P_{0}=\frac{1}{12} I_{200}$. The corresponding average estimation error $\mathbf{E}_{C K F}(k)$ is plotted in Fig. 3, from which it can been seen that under the BSCs the proposed CMHE has a better performance. As for the decentralized moving-horizon estimation (DMHE) problem, according to Algorithm 2, the optimal estimates can be derived and the corresponding average estimation error is shown in Fig. 3.

\section{CONCLUSION}

In this paper, the moving-horizon state estimation problems have been investigated for linear dynamic networks subject to binary encoding schemes. A novel model has been introduced to quantify the signal errors stemming from the communication processes, and it has been shown that the signal errors can 


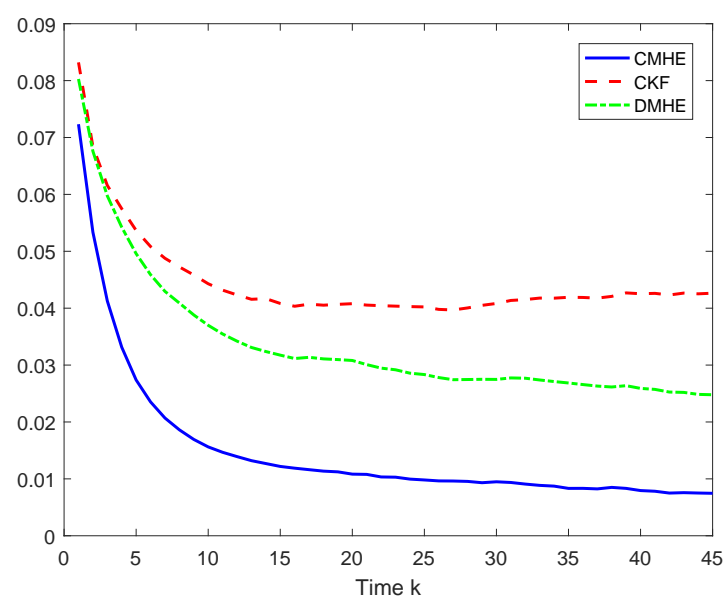

Fig. 3. The average estimation error of CMHE, CKF and DMHE.

be characterized by a cascade of a multiplicative and an additive white noise associated with the original signal. By solving two specified least-square optimization problems, we have respectively designed the centralized and decentralized movinghorizon estimators. Furthermore, sufficient criteria have been established to ensure stochastically ultimate boundedness of the proposed estimators with the binary encoding scheme. The feasibility and effectiveness of the proposed approaches have been verified via a numerical example.

\section{REFERENCES}

[1] A. Alessandri and M. Awawdeh, Moving-horizon estimation with guaranteed robustness for discrete-time linear systems and measurements subject to outliers, Automatica, vol. 67, pp. 85-93, 2016.

[2] A. Alessandri, M. Baglietto, and G. Battistelli, Receding-horizon estimation for discrete-time linear systems, IEEE Transactions on Automatic Control, vol. 48, no. 3, pp. 473-478, 2003.

[3] A. Bernstein, K. Steiglitz, and J. Hopcroft, Encoding of analog signals for binary symmetric channels, IEEE Transactions on Information Theory, vol. 12, no. 4, pp. 425-430, 1966.

[4] Y. Cui, Y. Liu, W. Zhang, T. Hayat, and F. E. Alsaadi, Sampled-data state estimation for a class of delayed complex networks via intermittent transmission, Neurocomputing, vol. 260, pp. 211-220, Oct 2017.

[5] X. Ge, Q. Han and F. Yang, Event-based set-membership leaderfollowing consensus of networked multi-agent systems subject to limited communication resources and unknown-but-bounded noise, IEEE Transactions on Industrial Electronics, Vol. 64, No. 6, pp. 5045-5054, Jun. 2017.

[6] Y. Guo and B. Huang, Moving horizon estimation for switching nonlinear systems, Automatica, vol. 49, no. 11, pp. 3270-3281, 2013

[7] J. Hu, Z. Wang, S. Liu, and H. Gao, A variance-constrained approach to recursive state estimation for time-varying complex networks with missing measurements, Automatica, vol. 64, pp. 155-162, 2016.

[8] R. Kalman, A new approach to linear filtering and prediction problems, Journal of basic Engineering, vol. 82, no. 1, pp. 35-45, 1960.

[9] U. A. Khan and J. M. F. Moura, Distributing the Kalman filter for largescale systems, IEEE Transactions on Signal Processing, vol. 56, no. 10, pp. 4919-4935, 2008.

[10] H. Leung, C. Seneviratne, and M. Xu, A novel statistical model for distributed estimation in wireless sensor networks, IEEE Transactions on Signal Processing, vol. 63, no. 12, pp. 3154-3164, 2015.

[11] L. Li, D. W. C. Ho and J. Lu, Event-based network consensus with communication delays, Nonlinear Dynamics, Vol. 87, No. 3, pp. $1847-$ 1858, Feb. 2017.

[12] W. Li, Y. Jia and J. Du, Variance-constrained state estimation for nonlinearly coupled complex networks, IEEE Transactions on Cybernetics, Vol. 48, No. 2, pp. 818-824, Feb. 2018.
[13] A. Liu, L. Yu, W. Zhang, and M. Chen, Moving horizon estimation for networked systems with quantized measurements and packet dropouts, IEEE Transactions on Circuits and Systems I: Regular Papers, vol. 60, no. 7, 1823-1834, 2013.

[14] D. Liu, Zidong Wang, Y. Liu and F. E. Alsaadi, Extended Kalman filtering subject to random transmission delays: dealing with packet disorders, Information Fusion, (ISSN 1566-2535) Vol. 60, Aug. 2020, pp. 80-86.

[15] Q. Liu, Z. Wang, X. He, and D. H. Zhou, On Kalman-consensus filtering with random link failures over sensor networks, IEEE Transactions on Automatic Control, vol. 63, no. 8, pp. 2701-2708, 2018.

[16] Q. Liu, Z. Wang, X. He, and D. H. Zhou, Event-triggered resilient filtering with measurement quantization and random sensor failures: Monotonicity and convergence, Automatica, vol. 94, pp. 458-464, 2018.

[17] S. Liu, Z. Wang, Y. Chen and G. Wei, Protocol-based unscented Kalman filtering in the presence of stochastic uncertainties, IEEE Transactions on Automatic Control, vol. 65, no. 3, pp. 1303-1309, Mar. 2020.

[18] J. Qin, M. Li, L. Shi and X. Yu, Optimal denial-of-service attack scheduling with energy constraint over packet-dropping networks, IEEE Transactions on Automatic Control, Vol. 63, No. 6, pp. 1648-1663, Jun. 2018.

[19] C. V. Rao, J. B. Rawlings, and J. H. Lee, Constrained linear state estimation-a moving horizon approach, Automatica, vol. 37, no. 10, pp. 1619-1628, 2001.

[20] C. V. Rao, J. B. Rawlings, and D. Q. Mayne, Constrained state estimation for nonlinear discrete-time systems: Stability and moving horizon approximations, IEEE Transactions on Automatic Control, vol. 48, no. 2, pp. 246-258, Feb. 2003.

[21] X. Ren, J. Wu, K. H. Johansson, G. Shi and L. Shi, Infinite horizon optimal transmission power control for remote state estimation over fading channels, IEEE Transactions on Automatic Control, Vol. 63, No. 1, pp. 85-100, Jan. 2018.

[22] Y. Shen, Z. Wang, B. Shen, F. E. Alsaadi and F. E. Alsaadi, Fusion estimation for multi-rate linear repetitive processes under weighted Try-Once-Discard protocol, Information Fusion, vol. 55, pp. 281-291, Mar. 2020.

[23] H. Su, Z. Rong, M. Z. Q. Chen, X. Wang, G. Chen, and H. Wang, Decentralized adaptive pinning control for cluster synchronization of complex dynamical networks, IEEE Transactions on Cybernetics, vol. 43, no. 1, pp. 394-399, 2013.

[24] L. Wang, Z. Wang, G. Wei and F. E. Alsaadi, Variance-constrained $H_{\infty}$ state estimation for time-varying multi-rate systems with redundant channels: The finite-horizon case, Information Sciences, vol. 501, pp. 222-235, Oct. 2019.

[25] Z. Wang, M. Andrews, Z. Wu, L. Wang and C. Bauch, Coupled diseasebehavior dynamics on complex networks: A review, Physics of Life Reviews, Vol. 15, pp. 1-29, Dec. 2015.

[26] W. Xu, D. W. C. Ho, J. Zhong and B. Chen, Distributed edge eventtriggered consensus protocol of multi-agent systems with communication buffer, International Journal of Robust and Nonlinear Control, Vol. 27, No. 3, pp. 483-496, Feb. 2017.

[27] Y. Xu, R. Lu, H. Peng, K. Xie and A. Xue, Asynchronous dissipative state estimation for stochastic complex networks with quantized jumping coupling and uncertain measurements, IEEE Transactions on Neural Networks and Learning Systems, Vol. 28, No. 2, pp. 268-277, Feb. 2017.

[28] B. Xue, S. Li, and Q. Zhu, Moving horizon state estimation for networked control systems with multiple packet dropouts, IEEE Transactions on Automatic Control, vol. 57, no. 9, pp. 2360-2366,2012.

[29] F. Yang, N. Xia and Q. Han, Event-based networked islanding detection for distributed solar pv generation systems, IEEE Transactions on Industrial Informatics, Vol. 13, No. 1, pp. 322-329, Feb. 2017.

[30] L. Zhang, Y. Zhu, and W. Zheng, State estimation of discrete-time switched neural networks with multiple communication channels, IEEE Transactions on Cybernetics, vol. 47, no. 4, pp. 1028-1040, 2017.

[31] X. Zhang and Q. Han, Event-triggered $H_{\infty}$ control for a class of nonlinear networked control systems using novel integral inequalities, International Journal of Robust and Nonlinear Control, Vol. 27, No. 4, pp. 679-700, Mar. 2017.

[32] L. Zou, Z. Wang, H. Gao, and X. Liu, Event-triggered state estimation for complex networks with mixed time delays via sampled data information: The continuous-time case, IEEE Transactions on Cybernetics, vol. 45, no. 12, pp. 2804-2815, 2015.

[33] M. Zorzi, Robust kalman filtering under model perturbations, IEEE Transactions on Automatic Control, vol. 62, no. 6, pp. 2902-2907, 2017.

[34] M. Zorzi, On the robustness of the Bayes and Wiener estimators under model uncertainty, Automatica, vol. 83, pp. 133-140, 2017. 